\title{
The Ultrastructure of the Spore of Nosema algerae (Protozoa, Microsporida), in Relation to the Hatching Mechanism of Microsporidian Spores
}

\author{
By R. E. SINDEN AND ELIZABETH U. CANNING \\ Department of Zoology, Imperial College, London $S_{7} W_{7} B B$.
}

(Received 29 July 1974)

\begin{abstract}
SUMMARY
The spore of Nosema algerae from the tissues of Anopheles stephensi has a wall consisting principally of electron-dense exospore and electron-lucent endospore. The polar filament, composed of two membranes around an amorphous or tubular core, described eleven coils in the posterior half of the spore and joined anteriorly with the umbrella-shaped polar sac. The polaroplast consisted of close-packed single membranes and sacs disposed around the anterior straight part of the filament. There was no posterior vacuole. There were two nuclei in a central area of cytoplasm containing abundant ribosomes and cysternae of endoplasmic reticulum. The cytoplasm beneath the plasmalemma and around the coils of the polar filament lacked ribosomes. Various hypotheses for filament extrusion are discussed in the light of the present observations. It is proposed that the positions of the outer membranes of the polar filament are inverted during extrusion and that the core becomes a coat to the filament. Some of the cytoplasm, together with the nuclei, passes through the filament and probably acquires a membrane from the sealed tip of the filament to become the sporoplasm. The polaroplast does not participate structurally in extension of the filament.
\end{abstract}

\section{INTRODUCTION}

Early studies by Krieg (1955) and Huger (1960) on the ultrastructure of the spores of Nosema melolonthae and $N$. locustae, respectively, established the basic components of the microsporidian spore, now known as the spore coat or wall, polar sac and polar cap, polar filament, polaroplast and 'sporoplasm' comprised of nucleus and cytoplasm. Additional studies on different genera have added to the knowledge of the internal structure and led to conflicting theories on morphogenesis and the hatching mechanism, proposed by Lom \& Vávra (1963), Sprague \& Vernick (1968), Lom (1972) and Weidner (1972).

Ultrastructural studies on spores hatching in tissue culture (Ishihara, I968; Weidner, 1972) have shown that the sporoplasms, when extruded, have normal nuclei and simple cytoplasmic components. Vávra \& Undeen (1970) showed a spore which had almost completely hatched in which the plasmalemma and peripheral cytoplasm apparently remained within the spore membrane, though no special mention was made of this fact. Thus any hatching mechanism must postulate an origin for the new plasmalemma which exists around the emerged sporoplasm.

Examination of favourable material of Nosema algerae spores embedded in glycol methacrylate has enabled us to add to the information on spore structure given by Canning $\&$ Sinden (1973), and to evaluate the various hypotheses of spore hatching mechanisms. 
METHODS

Mid-guts from Anopheles stephensi, infected with both Nosema algerae and Plasmodium yoelii nigeriensis, were dissected into $3 \%$ glutaraldehyde in $0 . \mathrm{I} \mathrm{M}$-phosphate buffer $\mathrm{pH} 7 \cdot 4$. They were then fixed overnight at $4{ }^{\circ} \mathrm{C}$ and, following three $\mathrm{I} \mathrm{h}$ washes in $0.15 \mathrm{M}$-phosphate

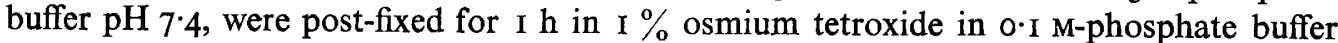
pH 7*4. The specimens were embedded in glycol methacrylate (Leduc \& Bernhard, 1967). Sections were stained in uranyl acetate and lead citrate. All micrographs were taken from material prepared for an autoradiographic study of the incorporation of $\left[{ }^{3} \mathrm{H}\right]$ adenosine (TRK 3; Radiochemical Centre, Amersham, Buckinghamshire).

\section{RESULTS}

Spore coat

(Figs. I, 2, 3, 4 and 7)

The cytoplasm was enveloped by a spore coat composed principally of a uniform electrondense exospore (ex), I00 nm thick, and an electron-lucent endospore (end). The latter was $200 \mathrm{~nm}$ thick except at the anterior end where it tapered to a narrow disc $20 \mathrm{~nm}$ thick overlying the polar sac. There was additionally a fine 9 to $20 \mathrm{~nm}$ electron-dense inner layer (ii) at the boundary of the endospore and cytoplasmic structures.

\section{Polar sac/polar filament complex}

(Figs. I, 2, 3, 4, 6 and 7)

Beneath a thin layer of cytoplasm $(l)$ at the anterior pole was the polar sac $(p s)$, an electrondense umbrella-shaped structure apparently bounded by a single membrane. This membrane was continuous with the outer membrane of the polar filament $(p f)$. Within the polar sac lay a denser mass, the polar cap $(p c)$, which separated the polar sac from the cavity of the polar filament.

The polar filament ran obliquely posteriad from the polar sac and swept outwards towards the peripheral cytoplasm; at about a third to a half of the way back, level with the anterior nucleus, it started to coil in the cytoplasm just within the plasmalemma. There were normally I I coils, which diminished in diameter from I $57 \mathrm{~nm}$ to less than $125 \mathrm{~nm}$ towards the posterior end. The coils were tilted at an acute angle to the longitudinal axis of the spore, and curved forward from the junction with the straight part of the filament with the result that some filament sections lay anterior to the nuclei. The posterior termination of the filament was not seen.

The filament contained an electron dense core $(c)$, which in the anterior coils might have been composed of about 12 tubules. At the anterior extremity of the filament the core became club-shaped and abutted on to the polar cap. Surrounding the core was an electronlucent tube which occasionally appeared to be subdivided into 14 to 20 sectors. The filament was bounded by two thick membranes of which the inner, often but not always, was of greater density. Whereas the outer membrane was continuous with that of the polar sac, the inner membrane terminated just short of the polar sac. 


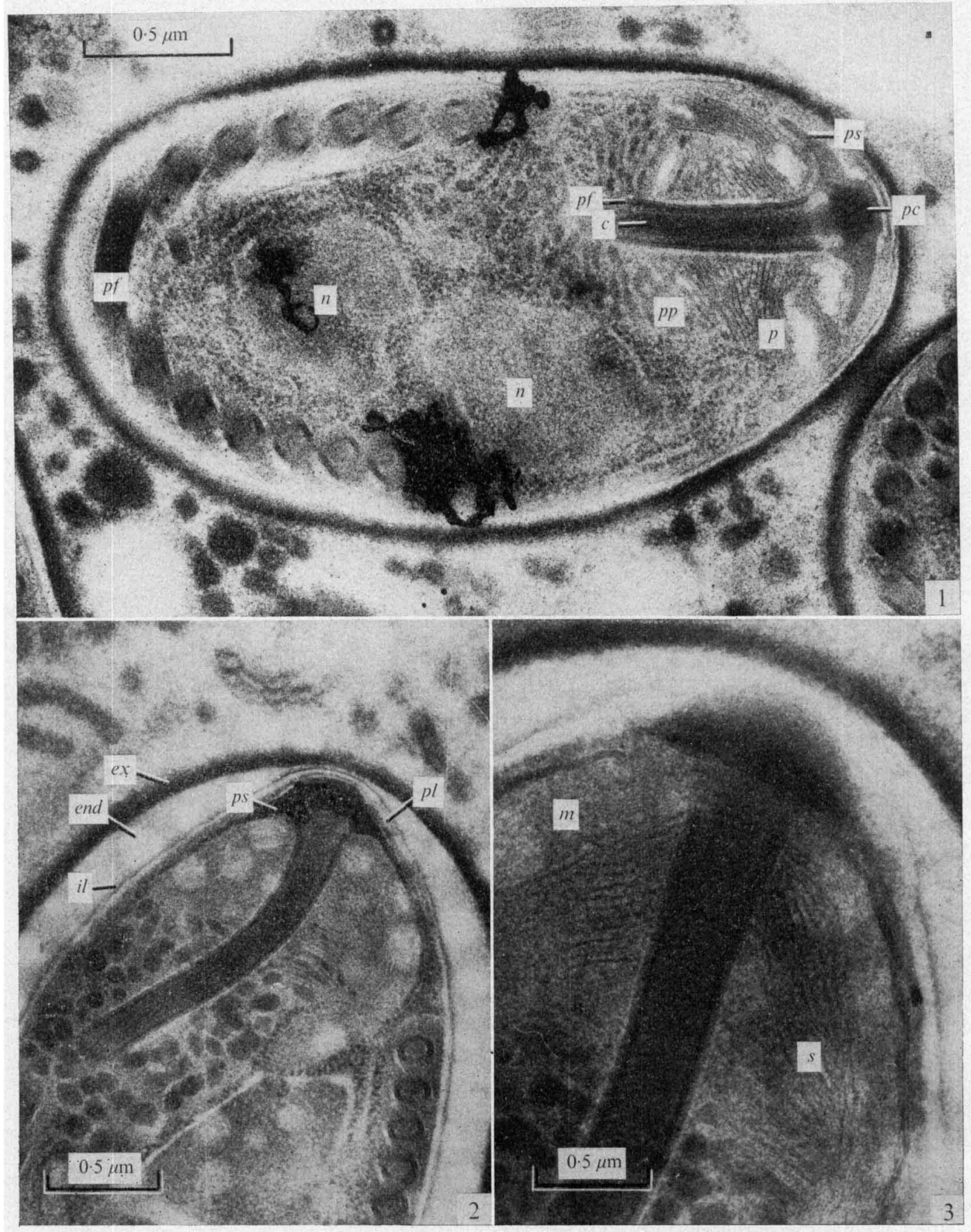

Figs. I to 3. Nosema algerae.

Fig. I. Longitudinal section of immature spore showing general relationships of nuclei $(n)$, polar sac $(p s)$, polar cap $(p c)$, polar filament $(p f)$ with core $(c)$ surrounded in its anterior region by polaroplast $(p)$ and polaroplast precursor $(p p)$.

Fig. 2. Longitudinal section of anterior region of mature spore illustrating components of spore wall [exospore $(e x)$, endospore $(e n d)$, electron-dense inner layer $(i l)$ ], plasmalemma $(p l)$ and narrow cytoplasmic layer covering anterior, free face of umbrella-shaped polar sac.

Fig. 3. Longitudinal section of anterior end of spore to show single membranes $(m)$ and sacs $(s)$ of the polaroplast. 

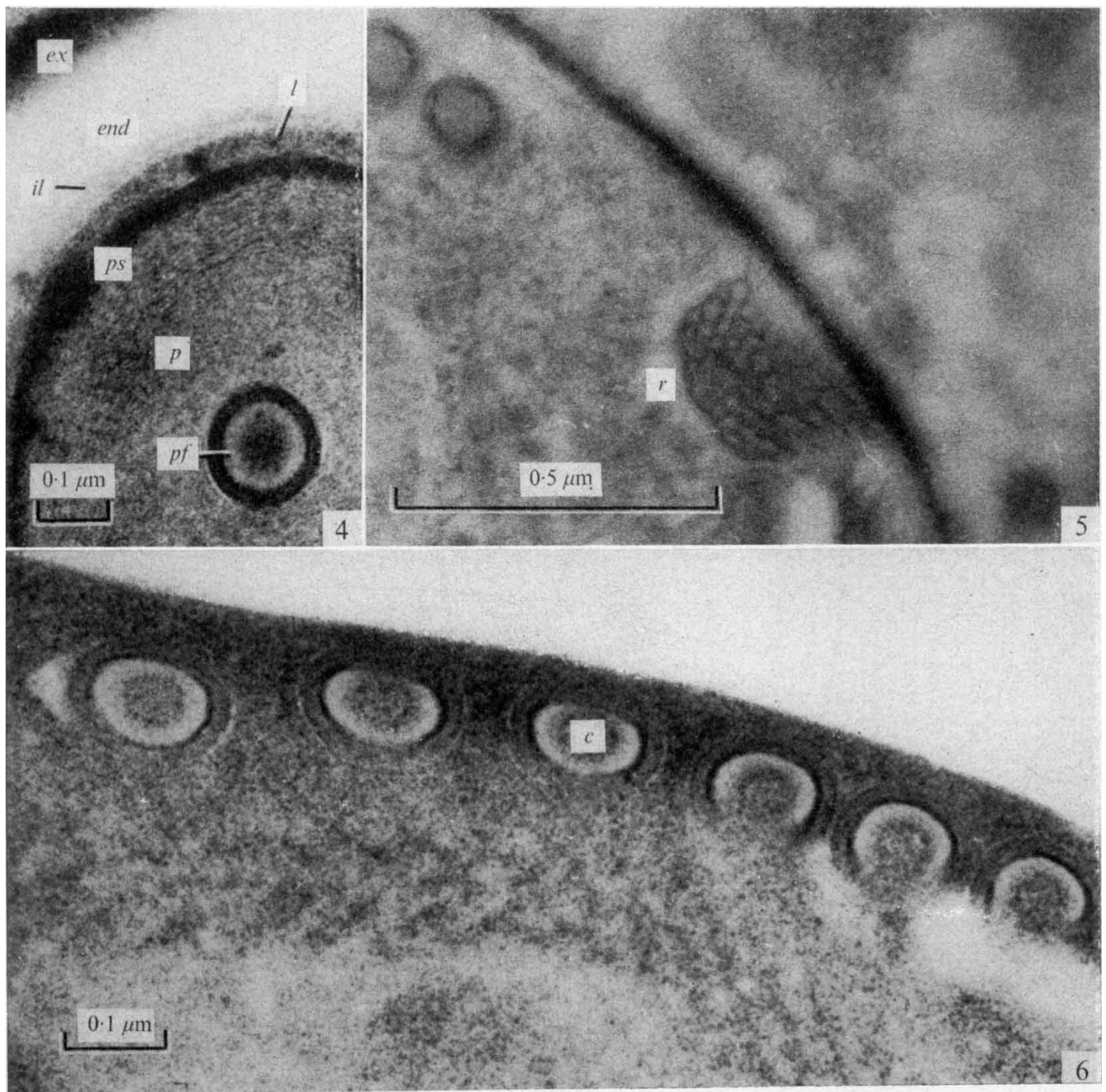

Figs. 4 to 6. Nosema algerae.

Fig. 4. Transverse section of anterior region of mature spore, showing polar filament $(p f)$, polaroplast $(p)$, polar sac $(p s)$, cytoplasmic layer $(l)$ and layers of the spore wall (ex, end, $i l)$.

Fig. 5. Section of reticular structure $(r)$ attached to plasmalemma of developing spore.

Fig. 6. Transverse section of coiled polar filament showing the two outer membranes and core (c). Some sections of filament show the tubular nature of the core.

\section{Polaroplast complex}

(Figs. I, 2, 3, 4 and 7)

The polaroplast $(p)$ was composed of a complex stack of membranous sheets which surrounded the anterior part of the filament within the recurved umbrella of the polar sac. The anterior lamellae were closely applied to the inner wall of the polar sac and occasionally appeared as single membranes $(m)$. These were stacked at an acute angle to the filament and enclosed the other membranes of the stack. Running posteriad the membranes, which were 


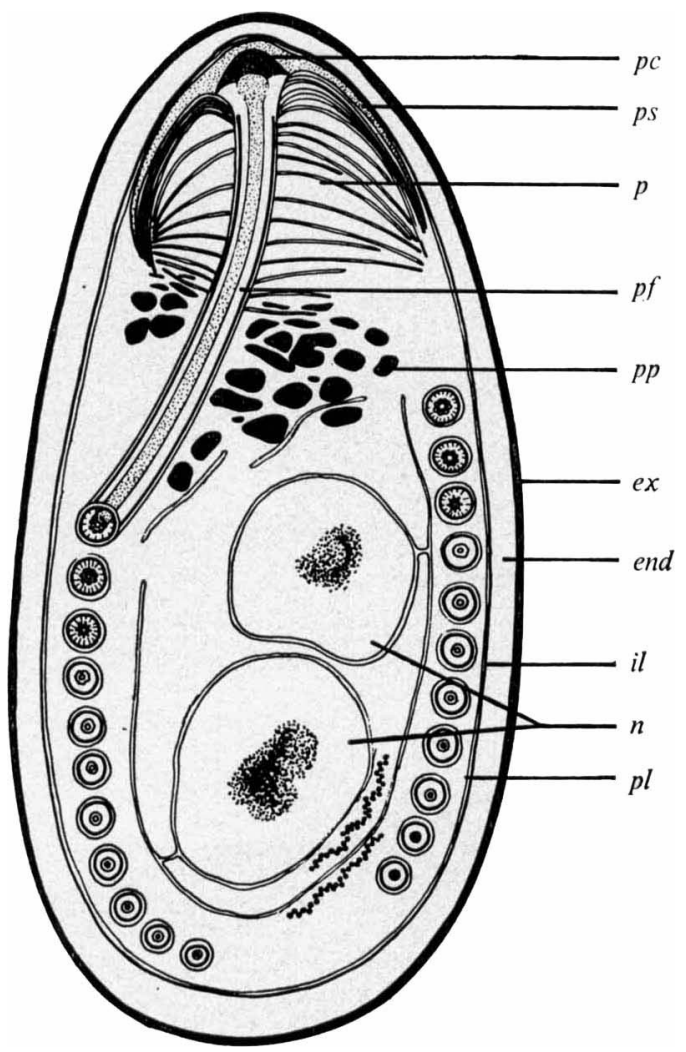

Fig. 7. Diagram of spore of Nosema algerae. For symbols see other Figures.

arranged as closed sacs $(s)$, sequentially became more perpendicular to the filament. Some posterior membranes were even at an obtuse angle to the filament.

Immediately posterior to the polaroplast lamellae was a region packed with electron-dense bodies $(p p)$ forming a reticulum, often appearing circular in section. They surrounded the filament as it swept outwards towards the peripheral cytoplasm. At their junction with the polaroplast they were flattened and interwoven with the membranes, a condition which suggested they were polaroplast precursors and not, as we have previously suggested (Canning \& Sinden, 1973), a filament-forming substance.

\section{Nuclei \\ (Figs. I and 7)}

The two nuclei $(n)$ lay closely apposed, one behind the other along the long axis of the spore. Each was surrounded by its own nuclear envelope. Chromatin masses were found within the nucleoplasm.

\section{Cytoplasm}

(Figs. $1,2,5$ and 7)

The cytoplasm was differentiated as an area surrounding the coiled filament, free of ribosomes but not physically separated from the remainder, and a central area containing numerous ribosomes, some of which were organized as large polysomes along extensive 
cysternae of endoplasmic reticulum. The endoplasmic reticulum frequently formed a large cup-like region of cytoplasm within which lay the two nuclei.

The cytoplasm was bounded by a plasmalemma $(p l)$ which lay immediately within the spore wall. Occasionally a space was seen between the plasmalemma and the spore wall, probably due to shrinkage of the cytoplasm during processing. In a few immature spores which had not yet developed the endospore layer, a reticular structure $(r)$, attached to the plasmalemma, extended into the cytoplasm. Some specimens suggested that this structure, whose function is unknown, existed as an oblique girdle around the spore. Filament-forming substance present at the posterior extremity of immature spores diminished in quantity in mature spores. A posterior vacuole was not seen, which accorded with the appearance of the spores in the light microscope (Canning \& Hulls, 1970; Vávra \& Undeen, 1970).

\section{DISCUSSION}

Several theories exist about the hatching mechanism of microsporidian spores (Lom \& Vávra, 1963; Sprague \& Vernick, 1968; Lom, 1972; Weidner, 1972). Recent ultrastructural evidence that the sporoplasm is a simple cell bounded by a plasmalemma and containing nuclei, membrane and ribosomes invalidates Sprague \& Vernick's (1968) hypothesis that the naked genome is inoculated into the host cell. This conclusion is supported by our finding that the two nuclei in the spore of Nosema algerae are each surrounded by their own membrane. An essential premise of the hatching mechanism proposed by Lom \& Vávra (1963), based on light microscopy, was that the sporoplasm existed within the spore as a separate entity and was surrounded by a plasmalemma while passing through the filament. Electron microscope studies do not substantiate this.

The theories of Lom \& Vávra (1963), Lom (1972) and Weidner (1972) share a common mechanism for filament extrusion - increased internal pressure resulting from swelling of the polaroplast and posterior vacuole. The ultrastructural studies of Lom (1972) on Nosema sp. and Ishihara (1968) on N. bombycis, together with the combined ultrastructural and cytochemical data of Weidner (1972) on N. michaelis and N. lophii, have clearly established that the filament is everted during extrusion. Despite this agreement the above authors differ considerably in their interpretation of other aspects of the hatching mechanism.

Lom (1972) suggested that the 'resting' polar filament contains three concentric osmiophilic layers, and lies within a membrane-limited cavity of the cytoplasm. The micrographs of Canning \& Sinden (1973), Gassouma \& Ellis (1973) and this study may be interpreted similarly. Lom (1972) suggested that the two outermost layers are membranes but the inner osmiophilic core is not. Our observations confirm that the core is not membranous but is either amorphous or composed of tubular structures. This layer cannot therefore become the outer membrane of the extruded filament as suggested tentatively by Lom (1972). The remaining two membranes could invert their positions on extrusion of the filament and the osmiophilic core could be discharged to form part of the glycoprotein coat found around the extruded filament by Weidner (1972). He described only a single membrane bounding the resting polar filament of $N$. michaelis and proposed that the additional membrane present round the extruded filament was derived from the polaroplast. However, his micrographs (Weidner, 1972, Fig. 4) suggest that two membranes may be present in the resting filament and therefore that the same mechanism of filament extrusion could apply to this as well as to other species.

Lom (1972, Fig. I2) illustrates a partially everted filament after negative staining, from which it appears that one or perhaps both of the filament membranes are sealed at the 
prospective filament tip. It is difficult to consider a mechanism of filament eversion based on hydrostatic pressure within the spore unless the filament is sealed at its tip. Further, the presence of a sealed filament provides a simple explanation for the source of the sporoplasm plasmalemma. The sporoplasm cannot be invested by the plasmalemma of the spore since electron micrographs of empty spores (Vávra \& Undeen, 1970; Weidner, 1972) show that this membrane remains in the spore following discharge of the sporoplasm. Weidner (1972) tentatively suggested that the sporoplasm might acquire a surface membrane by passing through the extruded filament between its two membranes, but micrographs of Ishihara (1968) and Lom (1972) are more indicative that the sporoplasm passes through the central lumen of the tube, considerably distending the tube as it passes. Since Ishihara (1968) has shown a remarkable similarity between the structure of the plasmalemma of the sporoplasm and the membrane of the filament and Weidner (1972) has shown the two to ke continuous, it is reasonable to suggest that the membranes of the sealed tube are distended around the sporoplasm when it reaches the tip of the filament.

To account for the apparent 2- to 3 -fold increase in length of the extruded polar filament compared with the 'resting' filament, Weidner (I972) proposed that the polaroplast membranes became incorporated into the extruded structure. Weaknesses in Weidner's hypothesis are that the filament would discharge through the region of highest pressure in the spore and that the filament would not itself evert. Furthermore, if the polaroplast membranes were incorporated into the wall of the tube, the wall would have to be highly folded. Though Lom (1972) showed convolutions on the surface of the everted filament these would not be sufficient to account for the extensive polaroplast membrane system. Should the increase in length of the filament upon extrusion prove to be a general phenomenon, a satisfactory mechanism by which this is achieved has yet to be found.

The hatching mechanisms described suggest that either the polaroplast or the posterior vacuole, or both, cause the increased pressure in the spore. We have been unable to find a posterior vacuole in $N$. algerae, and must assume therefore that all the pressure required for eversion of the filament is derived from the expansion of the sacs of the polaroplast.

This work was supported by a grant from the Medical Research Council. We gratefully acknowledge the technical assistance of Mrs B. Spain and J. P. Nicholas.

\section{REFERENCES}

Canning, E. U. \& Hulls, R. H. (1970). A microsporidian infection of Anopheles gambiae Giles from Tanzania, interpretation of its mode of transmission and notes on Nosema infections in mosquitoes. Journal of Protozoology 17, 53 I-539.

CANNING, E. U. \& Sinden, R. E. (1973). Ultrastructural observations on the development of Nosema algerae Vávra and Undeen (Microsporida, Nosematidae) in the mosquito Anopheles stephensi Liston. Protistologica 9, 405-415.

Gassouma, M. S. S. \& Ellis, D. S. (1973). The ultrastructure of sporogonic stages and spores of Thelohania and Plistophora (Microsporida, Nosematidae) from Simulium ornatum larvae. Journal of General Microbiology 74, 33-43.

Huger, A. (1960). Electron microscope study on the cytology of a microsporidian spore by means of ultrathin sectioning. Journal of Insect Pathology 2, 84-105.

IsHIHARA, R. (I968). Some observations on the fine structure of sporoplasm discharged from spores of a microsporidian, Nosema bombycis. Journal of Invertebrate Pathology 12, 245-258.

KRIEG, A. (1955). Über Infektionskrankheiten bei Engerlingen von Melolontha spec. unter besonderer Berücksichtigung einer Mikrosporidien-Erkrankung. Zentralblatt für Bakteriologie, Parasitenkunde, Infektionskrankheiten und Hygiene ro8, 535-538. 
LeDUC, E. H. \& BERNHARD, W. (I967). Recent modification of the glycol methacrylate embedding procedure. Journal of Ultrastructure Research 19, 196-199.

LoM, J. (1972). On the structure of the extruded microsporidian filament. Zeitschrift für Parasitenkunde $\mathbf{3 8}^{8}$, 200-213.

LoM, J. \& VÁVRA, J. (1963). The mode of sporoplasm extrusion in microsporidian spores. Acta protozoologica I, 8I-90.

SpRaGUe, V. \& Vernick, S. H. (I968). Light and electron microscope study of a new species of Glugea (Microsporida, Nosematidae) in the 4-spined stickleback Apeltes quadracus. Journal of Protozoology $\mathbf{1 5}$, 547-57I.

VÁvra, J. \& UndeEn, A. H. (1970). Nosema algerae n.sp. (Cnidosporida, Microsporida), a pathogen in a laboratory colony of Anopheles stephensi Liston (Diptera, Culicidae). Journal of Protozoology 17, 240-249.

WEIDNER, E. (1972). Ultrastructural study of microsporidian invasion into cells. Zeitschrift für Parasitenkunde 40, 227-242. 